\title{
Optical algal biosensor using alkalìne phosphatase for determination of heavy metals
}

\section{Claude Durrieu(1)*, Canh Tran-Minh(2)}

(1) Ecole Nationale des Travaux Publics del'Etat, Laboratoire des Sciences de l'Environnement, 3, Rue Maurice Audin, 69518 Vaulx en Velin cedex, France

(2) Ecole Nationale Supérieure des Mines de Saint Etienne, Centre SPIN ; Département PMMC ; 158 Cours Fauriel ; 42023 Saint-Étienne Cedex 2, France

\begin{abstract}
A biosensor is constructed to detect heavy metals from inhibition of alkaline phosphatase (AP) present on the external membrane of Chlorella vulgaris microalgae. The microalgal cells are immobilized on removable membranes placed in front of the tip of an optical fiber bundle inside a homemade microcell. C. vulgaris was cultivated in the laboratory and its alkaline phosphatase activity is strongly inhibited in the presence of heavy metals. This property has been used for the determination of those toxic compounds.
\end{abstract}

Keywords:

biosensor; algae; Chlorella; alkaline phosphatase; fluorescence; heavy metals; ecotoxicology.

\section{Introduction}

It is known that heavymeta ls have a toxic effect on living organisms through their action on enzymes of the metabolic chain. The discharge of those compounds into nature leads to the requirement for methods that can monitor them in low concentrations for their control in the environment (Gu et al., 1999; Schubnell et al., 1999).

Heavy metals are usually determined by sophisticated techniques like ionic chromatography, mass spectrometry, inductively coup led plasma, and polarography, which need to be carried out in laboratories after sampling. These methods are not suitable for continuous monitoring in the environment (Rogers and Gerlach, 1999). Heavy metals can also be determined with ion-sensitive electrodes; however, the electrode selectivity and response cannot be associated with the toxicity impact of those chemicals. Therefore, enzyme sensors have been studied and constructed for determination of inhibitors (Gayet et al., 1993; Cowell et al., 1995); they are based essentially on the inhibition of purified enzymes like urease, cholinesterase, and cytochrome oxidase (Tran-Minh, 1985; Albery et al., 1990). To provide inexpensive biosensors for environmental control, the enzyme purification step needs to be avoided and enzymes are kept as such in their natural system to ensure a better lifetime (Samson and Popovic, 1988; Rechnitz and Ho, 1990). This approach was adopted with the use of alkaline phosphatase located on the external membrane of the whole cell Chlorella vulgaris for direct determination of heavy metals. On the other hand, this enzyme, which plays a key role in phosphate metabolism, is easily accessible to pollutants since it is attached to the outer part of the cell membrane. In vivo inhibition of this enzyme reflects the natural deletorious effect of toxicants on microalgae and represents a real ecological interest considering that algae are involved in the primary step of the food chain.

\footnotetext{
${ }^{*}$ Corresponding author: durrieu@entpe.fr
} 


\section{Material and Methods}

\section{II.1. Algal Membrane Preparation}

The Chlorella vulgaris strain (CCAP 211/ 12) was purchased from The Culture Collection of Algae and Protozoa at Cumbria, United Kingdom. The axenic algal strain was grown in the culture medium and under conditions described by the International Organization for Standardization (ISO, 1989). To induce a high alkaline phosphatase concentration in the algal cell, the algal solution was centrifuged and the pellets were resuspended in phosphate-free medium to starve the algal cells for 25 days which corresponds to maximal enzyme activity. This resuspension was carried out under the same conditions and with the same culture medium as that used previously for the growth of this strain except there were no phosphate ions. Although the medium did not contain any phosphate, an increase in cell density was observed, particularly at the beginning of the subculture period, due to the consumption of the phosphate remaining in the cell. This phosphate consumption induces an increase in alkaline phosphatase specific activity. Two milliliters of the resulting aliquot was then filtered on a glass microfiber filter (GF/ C Whatman, 45.7-mm filter diameter, 1.2-mm pore diameter). Punching this filter provides small disks (8mm in diameter) which can be fitted to the optical transducer later.

A value of $0.73 \mathrm{~g} / \mathrm{L}$ dry wt was obtained after the filter had been dried for $2 \mathrm{~h}$ at 1051C and placed in a desiccator for $0.5 \mathrm{~h}$.

\section{II.2. Operating Principle}

Determination of alkaline phosphatase (AP) activity is carried out with methylumbelliferoyl phosphate (MUP) as substrate dissolved at various concentrations in a Tris- $\mathrm{HCl}(0.1 \mathrm{M}, \mathrm{pH}$ 8.5) buffer solution containing $1 \mathrm{mM} \mathrm{MgCl}_{2}$. This solution is referred to as MUP solution hereafter. MUP was purchased from Sigma; other chemicals from Merck.

The reaction product methylumbelliferone (MUF) is fluorescent:

$$
M U P \stackrel{A P}{\longrightarrow} M U F(\text { fluorescent })+\text { phosphate }
$$

AP activity can easily be measured from the MUF fluorescence emission (460nm wavelength) under excitation light (350nm wavelength) when the MUP solution is brought into contact with AP. This can be done either by adding the MUP solution into the microplate wells containing C. vulgaris or by injecting the MUP solution into the carrier stream leading to an algal biosensor. In the latter case, an optical fiber is required to send the excitation light to the algal membrane and to collect the fluorescence radiation up to the fluorometer. Since flow injection analysis (FIA) technique is involved, AP activity is determined from peak area (or peak height) corresponding to the concentration of substrate injected. For inhibitor determination, a given amount of heavy metal is introduced into the MUP solution to have the required concentration. A reduction in peak area or peak height is observed with use of the inhibitor solution.

\section{II.3. Construction of the Algal Optical Biosensor}

The biosensor was constructed with a removable algal membrane placed in a 1-mL homemade flow cell (Naessens et al., 2000). Opposite the membrane, a bifurcated bundle of randomized optical fibers was oriented so that the incident light hit the upper part of the membrane and the resulting fluorescence could be collected by the corresponding fibers and transmitted to the Spex Fluorolog 2 fluorometer (from J obin-Yvon) equipped with a microcomputer for data recording. This device was quite suitable for the assay under FIA conditions which require a very small amount of substrate for measurement of enzyme activity.

\section{Results}

\section{III.1. Induction of Alkaline Phosphatase}

AP activity in algal cells can be increased by inducing its production with the starvation method. This activity is measured with a batch system under air-saturated conditions. Enzyme activity was plotted as a function of time during the starvation period to assess the 
maximal specific activity(Figure 1). This sigmoid curve shows that at least 25 days of subculture is required to attain maximal activity. This time was used in our experiments.

\section{III.2. Effect of Substrate Concentration}

The configuration of the FIA manifold used is shown in Figure 2. AP activity of algae immobilized on the biosensor is determined with different substrate concentrations. Two substrates can be used: p-nitrophenyl phosphate (pNPP) and MUP. The former produces pnitrophenol, which absorbs at $405 \mathrm{~nm}$, and the latter gives MUF, which fluoresces at $460 \mathrm{~nm}$. The second substrate was chosen for its higher sensitivity and its simplicity in biosensor design. The substrate MUP was placed in a Tris buffer solution $(0.1 \mathrm{M}, \mathrm{pH}$ 8.5) containing $1 \mathrm{mM} \mathrm{MgCl} 2$ as enzyme activator, and AP activity was measured in terms of fluorescence intensity from the biosensor response. Various substrate volumes to be injected in the carrier stream were tested: a volume of $0.5 \mathrm{~mL}$ represents a good compromise to achieve an acceptable response time and good repeatability. A flow rate of $8.4 \mathrm{~mL} . \mathrm{min}-1$ was also used for the same reasons.

Figure 3 illustrates typical Michaelis-Menten behavior, and an approximate value of 20 mMMUP for the Michaelis constant is obtained from the curve.

\section{III.3. Determination of Heavy Metals}

The effect of toxic chemicals on AP was tested with cadmium and lead as heavy metals. Enzyme activity was determined from the fluorescence measurements in the presence and absence of metal in the $0.1 \mathrm{mM}$ MUP solution which nearly corresponds to the maximal velocity of the enzyme. When the substrate solution containing the metal is injected, the enzyme is inhibited, and peak area and peak height are reduced. A percentage of enzyme inhibition is introduced to emphasize the biosensor response to heavy metals as inhibitors while reducing experimental variations. It is defined as a ratio of the biosensor response in the presence of a heavy metal to the response in the absence of inhibitor. These responses can be compared with the results obtained with a microplate reader (Figure 4).

\section{Discussion}

The responses of the algal biosensor using immobilized alkaline phosphatase to cadmium and lead are in agreement with the results obtained with a microplate reader based on the inhibition of the same enzyme system. This means that the technique can be extended to other heavy metals such as nickel, mercury, copper, zinc, and chromium that inhibit algal alkaline phosphatase.

Heavy metals also inhibit algal growth (Vasseur and Pandard, 1988) but this process was not taken into account in these measurements because in this enzyme inhibition based method, determination of peak areas of peak heights in the presence and absence of heavy metals was carried out with a single algal membrane and consequently with the same amount of algae. Therefore, only specific AP activity of Chlorella is concerned in the biosensor response.

In addition, use of the percentage inhibition provides a reliable response that does not depend on algal strains. It also reduces the effect of long-term enzyme activity loss. Alkaline phosphatase was induced to have maximal activity: this procedure allows the use of a smaller amount of $\mathrm{C}$. vulgaris and the deposition of a thinner layer, which favors a fast response time. However, an optimal enzyme concentration is needed to improve the inhibitors detection limits (Tran-Minh, 1985).

The range of toxic concentrations detected with this biosensor makes it more suitable for determination of heavy metals present in waste water rather than surface water. Other experiments conducted in our laboratory have shown that this enzyme sensor is not sensitive to herbicides because AP is not inhibited by these compounds. Therefore, inhibition of algal photosynthesis cannot be assessed with this enzymatic technique. When herbicides are present together with heavy metals, only the latter is detected with this biosensor.

The biosensor response also depends on substrate concentration for a given concentration of heavy metal used as inhibitor. High substrate concentrations corresponding to the maximal rate of the enzyme often reduce enzyme dependence on substrate concentration. However, 
those concentrations cannot be used for enzymes that are inhibited by an excess of substrate, which interferes with the metal detection also based on enzyme inhibition. An optimal substrate concentration should then be determined from the activity- substrate curve.

Flow injection analysis associated with a biosensor provides repeatable results along with a low consumption of substrate. The system can easily be automated for the biosensor to operate in continuous monitoring.

\section{Conclusion}

Biosensors using immobilized enzymes present in whole cells have potential advantages for determination of toxic compounds as AP inhibitors. The enzyme remains in its natural environment which favors long-term stability and reflects the mechanism of toxic inhibition, being therefore of ecological interest. Biosensors based on inhibition of algal alkaline phosphatase are sensitive to heavy metals; they are complements to those based on photosynthesis inhibition designed for herbicide determination (Naessens et al., 2000).

\section{References}

Albery, W. J ., Cass, A. E. G., and Shu, Z. X. (1990). Inhibited enzyme electrodes. Part 2: The kinetics of the cytochrome oxidase system. Biosens. Bioelectron. 5, 379- 395.

Cowell, D. C., Dowman, A. A., and Ashcroft, T. (1995). The detection and identification of metal and organic pollutants in potable water using enzyme assays suitable for sensor development. Biosens. Bioelectron. 10, 509- 516.

Gayet, J. C., Haouz, A., Geloso-Meyer, A., and Burstein, C. (1993). Detection of heavymetal salts with biosensors built with an oxygen electrode coupled to various immobilised oxidases and dehydrogenases. Biosens. Bioelectron. 8, 177- 183.

Gu, M. B., Gil, G. C., and Kim, J. H. (1999). A two stage minibioreactor system for continuous toxicity monitoring. Biosens. Bioelectron. 14, 355- 361.

International Organization for Standardization (1989). ISO 8692: Water Quality: Fresh Water Algal Growth Inhibition Test with Scenedesmus subspitacus and Selenestrum capricornutum.

Naessens, M., Leclerc J . C., and Tran-Minh, C. (2000). Fiber optic biosensor using Chlorella vulgaris for determination of toxic compounds. Ecotoxicol. Environ. Saf. 46, 181- 185.

Rechnitz, G. A., and Ho, M. Y. (1990). Biosensors based on cell and tissue material. J . Biotechnol. 15, 201- 218.

Rogers, K. M., and Gerlach, C. L. (1999). Update on environmental biosensors. Environ. Sci. Technol./ News, 498- 504.

Samson, G., and Popovic, R. (1988). Use of algal fluorescence for determination of phytotoxicity of heavy metals and pesticides as environmental pollutants. Ecotoxicol. Environ. Saf. 16, 272- 278.

Schubnell, D., Lehmann, M., Baumann, W., Root, F. G., Wolf, B., and Beck, C. F. (1999). An ISFET-algal (Chlamydomonas) hybrid provides a system for eco-toxicological tests. Biosens. Bioelectron. 14, 465-472.

Tran-Minh, C. (1985). Immobilized enzyme probes for determining inhibitors. Ion Selective Electrode Rev. 7, 41- 75.

Vasseur, P., and Pandard, P. (1988). Influence of some experimental factors on metal toxicity to Selenastrum capricornutum. Toxic. Assess. 3, 331- 343. 


\section{Figures}

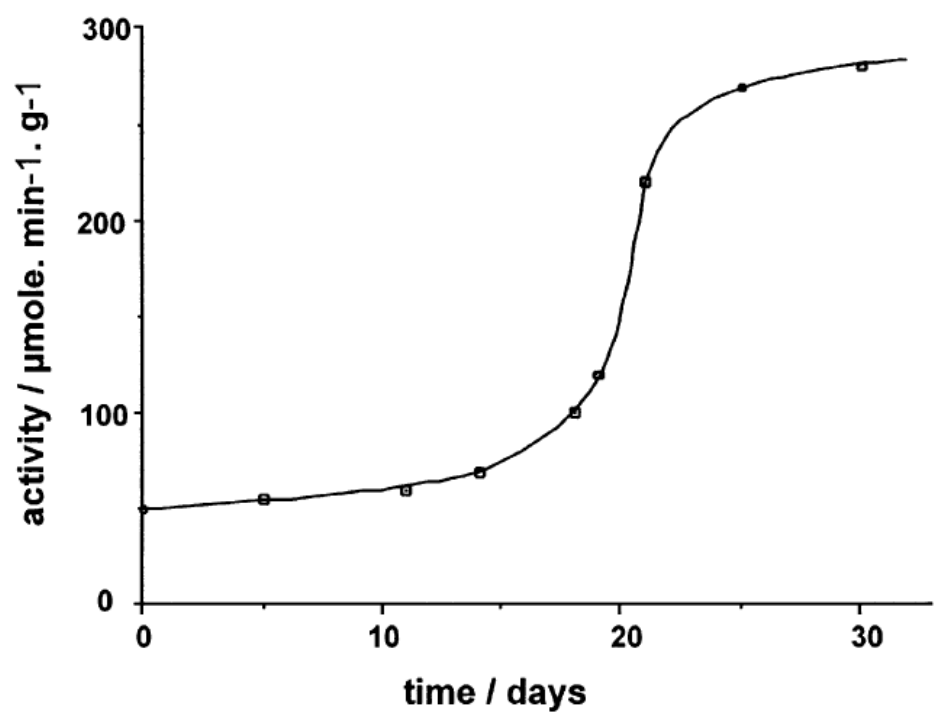

Figure 1: Induction of algal alkaline phosphatase activity using the starvation method.

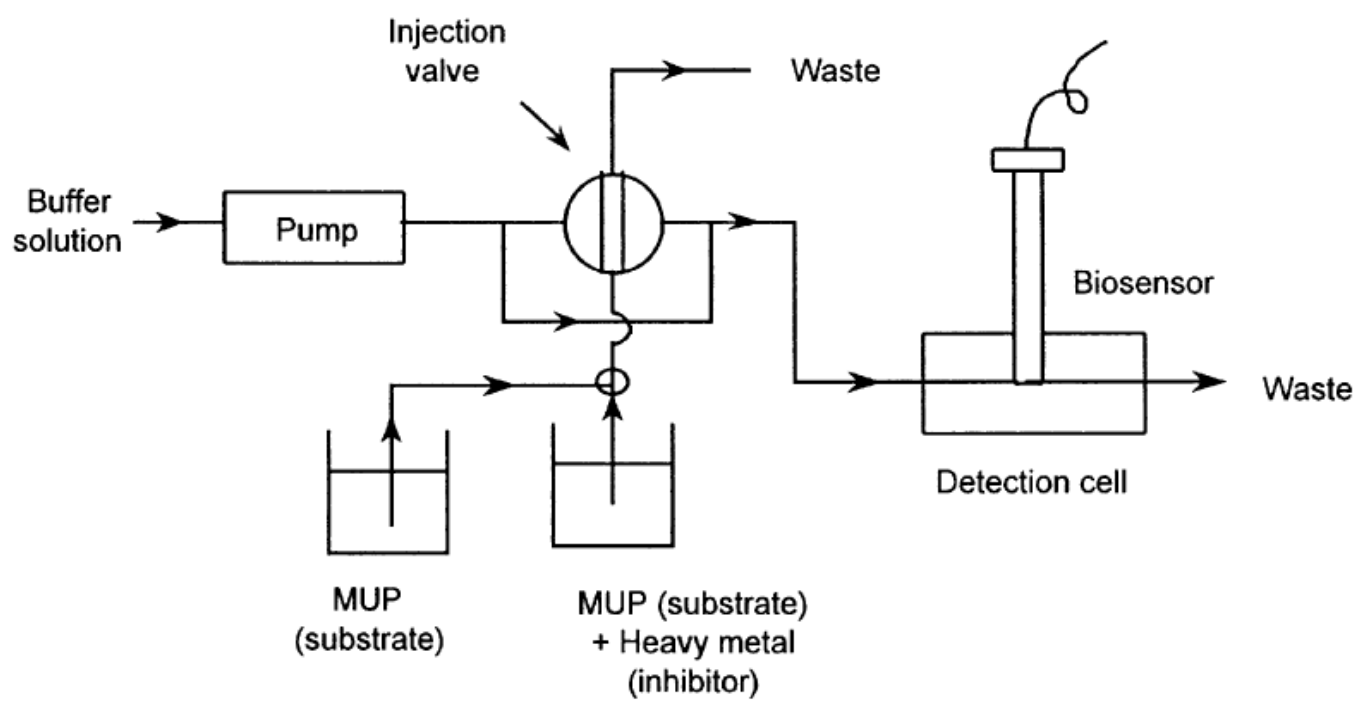

Figure 2: FIA manifold using an algal biosensor for determination of algal alkaline phosphatase activity. 
Talanta, 2003, 59(3), 535-44, doi: 10.1016/S0039-9140(02)00540-4

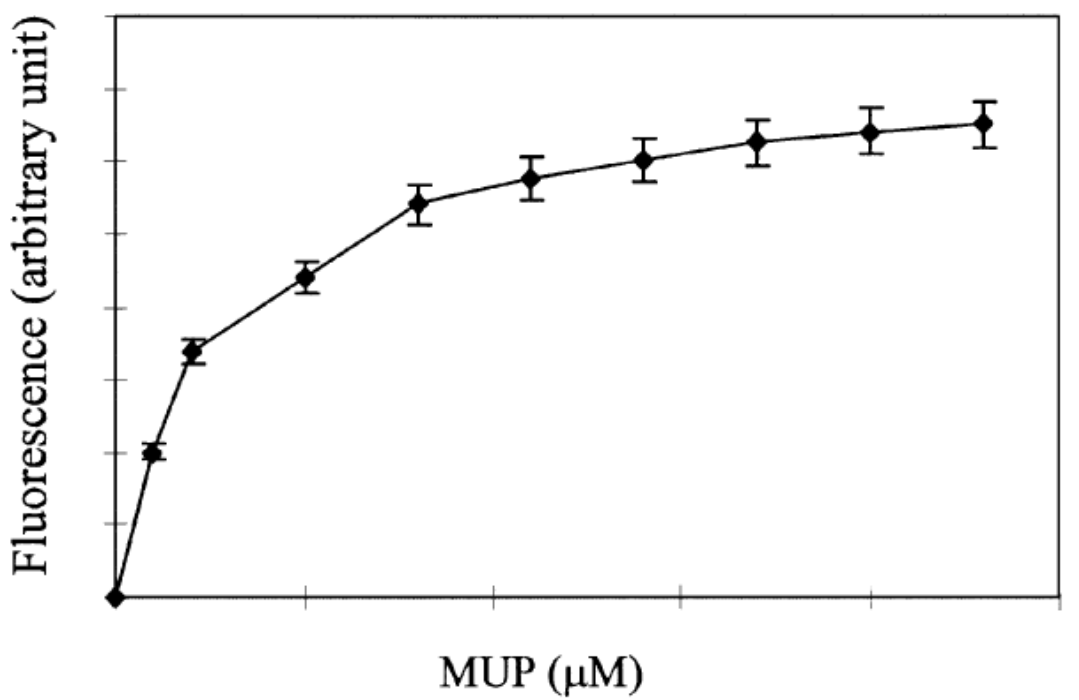

Figure 3: Response of an algal alkaline phosphatase biosensor as a function of substrate concentration.

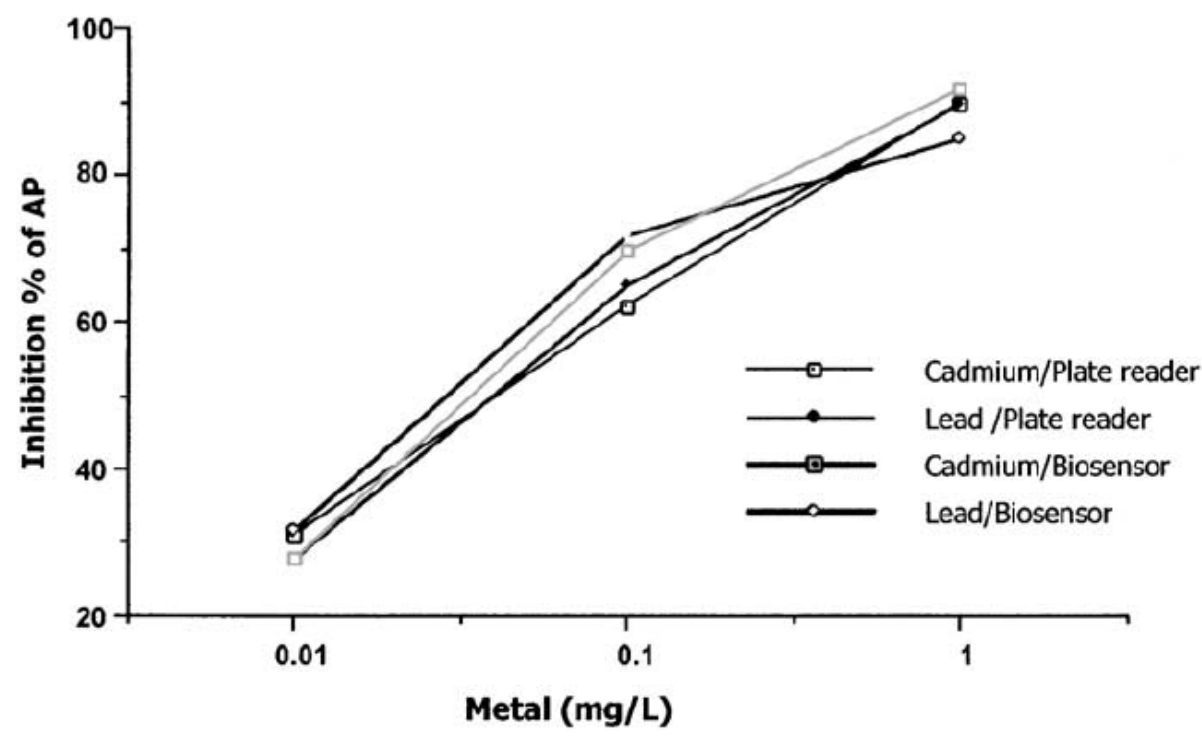

Figure 4: Comparison between microplate reader and biosensor responses to cadmium and lead concentrations in terms of percentage of inhibition of alkaline phosphatase (AP). 